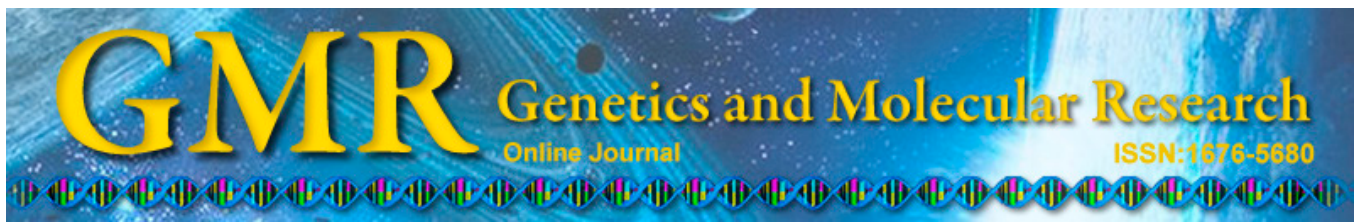

Short Communication

\title{
Cloning and characterization of up-regulated HbSINA4 gene induced by drought stress in Tibetan hulless barley
}

\author{
H.J. Yuan ${ }^{1,2}$, X.M. Luo ${ }^{3}$, T.S. Nyima ${ }^{1,2}$, Y.L. Wang ${ }^{1,2}$, Q.J. Xu ${ }^{1,2}$ and \\ X.Q. Zeng ${ }^{1,2}$ \\ ${ }^{1}$ Tibet Academy of Agriculture and Animal Husbandry Sciences, Lhasa, \\ Tibet, China \\ ${ }^{2}$ Barley Improvement and Yak Breeding Key Laboratory of Tibet Autonomous \\ Region, Lhasa, China \\ ${ }^{3}$ College of Forestry, Sichuan Agricultural University, Wenjiang, Sichuan, China \\ Corresponding author: X.Q. Zeng \\ E-mail: xingquanz_2@126.com \\ Genet. Mol. Res. 14 (4): 15312-15319 (2015) \\ Received October 30, 2014 \\ Accepted April 17, 2015 \\ Published November 30, 2015 \\ DOI http://dx.doi.org/10.4238/2015.November.30.7
}

ABSTRACT. Hulless barley is an important crop cereal in Tibetan, China. Drought is a major abiotic stress in barley production. In this study, we cloned the drought-related HbSINA4 gene from the variety 'Himalaya 10' and analyzed its expression patterns under different drought and rehydration conditions. The cDNA of HbSINA4 was 1052 bp long, including an open reading frame of $771 \mathrm{bp}$ that encoded a protein of 256 amino acids. The molecular weight of HbSINA4 protein was predicted to be $29.53 \mathrm{kDa}$ and the theoretical pI was 8.32 . Bioinformatic analysis showed that the HbSINA4 gene contained a protein kinase domain profile family signature motif, with high similarity to that of Oryza sativa and Brachypodium distachyon. Real-time polymerase chain reaction (PCR) assays revealed that gene 
expression declined rapidly with increasing drought stress; in contrast, its expression increased after rehydration treatment. Therefore, the HbSINA4 gene responds to the drought stress and plays an important role in barely drought resistance. Furthermore, our results provide information which may be useful in other temperate crop studies and in aiding resistance to drought.

Key words: Barley; Drought stress; Gene cloning; Gene expression; HbSINA4; Hordeum vulgare

\section{INTRODUCTION}

Because of increased global warming, drought stress has threatened the world's food security. Additionally, the world human population is predicted to reach 9.4 billion by 2050 , as predicted by the UN, and more food will be required to feed this large number of people. Drought stress is one of the more important environmental stresses affecting agricultural productivity worldwide (Boyer, 1982). In crops, drought can affect vegetative growth and yield formation at any developmental stage depending on the cropping region. Severe drought stress can even cause plant death (Zhao and $\mathrm{Li}, 1999)$. To face these challenges, plant breeders and geneticists are seeking strategies to overcome or reduce the detrimental effects of drought. Drought-resistant varieties have been developed in many breeding programs. To achieve this, the first step is to understand the biological processes and genetic basis of plant responses to drought.

The drought resistance of crops is controlled by multiple genes, including those involved in signal transduction and transcriptional regulation, as well as the expression of genes involved in plant protection, defense, and resistance stresses (Wang et al., 2003). Drought resistance is closely related to a range of biological process, such as osmotic regulation, autoxidation, photosynthesis, plant hormones, and dehydration-protection protein metabolism, indicating that the response is complex (Wang, 2013). Numerous physiological and biochemical indices, methods, and technologies to combat drought stress have been developed in previous studies (Singh et al., 1972; Acar et al., 2001). Several genes were identified to be induced by drought (Diab et al., 2004; Guo et al., 2009; Zhang et al., 2009). Quantitative trait loci mapping has been used in Arabidopsis thaliana, Triticum aestivum, and Oryza sativa, among others (Zhao et al., 2005; Wang, 2007; Nie, 2009). Based on chlorophyll a fluorescence OLKJIP under drought stress and re-watering, Oukarroum et al. (2007) evaluated chlorophyll content and fluorescence parameters as indicators of drought tolerance in barley. Barley is one of the most important cereal crops in many countries, including China. In most countries, barley is often the only possible rain-fed crop that farmers can grow, and is often subjected to extreme water deficit during the dry season. In Tibet, the early stage of highland barley growth is typically threatened by drought. In order to improve the yield and quality of highland barley, it is crucial to select drought-resistant elite varieties, which can be developed by understanding the genetic mechanisms of drought resistance.

The seven in absentia (SINA) protein belongs to the ubiquitin ligase E3 family. SINA protein plays important roles in many biological processes through ubiquitination of specific proteins. Little is known about the function of SINA family in plant, with a few exceptions. For example, SINAT5 has been found to be related to lateral root formation in A. thaliana (Xie et al., 2002). Additionally, Welsch et al. (2007) reported that an interaction between SINAT2 
and transcription factor AtRAP2.2 are involved in the formation of carotene. Den Herder et al. (2008) found that SINA protein can affect plant growth and nodule formation. In this study, we cloned the SINA gene, which is related to resistance to drought, and then analyzed its expression patterns under drought stress.

\section{MATERIAL AND METHODS}

\section{Plant materials and drought treatments}

An elite hulless barley cultivar 'Himalaya 10', which shows good drought tolerance, was used. This cultivar was kindly provided by Tibetan Academy of Agriculture and Animal Husbandry Sciences. Soil used for growing barley was measured for water content, and the moisture was adjusted to $33.4 \%$ by adding water. Seedlings of Himalaya 10 were grown in a greenhouse under identical conditions at a temperature of $23^{\circ} / 15^{\circ} \mathrm{C}$ (day/night) and relative humidity of $10-20 \%$. Prior to drought stress treatment, seedlings were watered every 2 days to maintain the relative soil moisture content (RSMC) at 33.4\%. The drought stress began when the seedlings grew to 2-and-a-half leaf stage (18 days after sowing). Drought stress included 6 different levels by limiting the water supply, with RSMC values of 33.4, 27.5, 21.1, 15.5, 9.8 , and $4.8 \%$. At each RSMC level, leaf samples were harvested. After drought stress, the remaining seedlings at $4.8 \%$ RSMC were rewatered to restore the RSMC to $33.4 \%$, and the leaf samples since rehydration (2, 4, and $8 \mathrm{~h}$ after RSMC 33.4\%) were collected. All leaf samples were immediately frozen in liquid nitrogen and stored at $-80^{\circ} \mathrm{C}$ until RNA extraction.

\section{Cloning of cDNA of HbSINA4}

Total RNA was extracted from the leaf samples and treated with DNase I. Primers were produced using Primer Premier 5.0 based on the sequences from the transcriptome sequencing analysis results (data not shown). Total RNA was reversely transcribed into cDNA. The primers used were A1-FP (AGGTACCATGTACCCTCCGATCCACCAG) and A1-RP (AACTAGTTCAGCTGAAGAGATTAGGGATGC). Real-time polymerase chain reaction (RT-PCR, S-1000 Thermal Cycler, Bio-Rad, Hercules, CA, USA) amplification was carried out in a $20-\mu \mathrm{L}$ volume containing $0.2 \mu \mathrm{L} 5 \mathrm{U} / \mu \mathrm{L}$ Taq DNA polymerase, $2 \mu \mathrm{L} 10 \mathrm{X}$ PCR buffer, $1.6 \mu \mathrm{L} 10 \mathrm{mM}$ dNTP mixture, $0.8 \mu \mathrm{L} 10 \mu \mathrm{M}$ of each primer, and distilled water. After complete denaturation of the template DNA at $94^{\circ} \mathrm{C}$ for $4 \mathrm{~min}$, PCR was performed with 34 cycles of $50 \mathrm{~s}$ at $94^{\circ} \mathrm{C}, 45 \mathrm{~s}$ at $51{ }^{\circ} \mathrm{C}$, and $1 \mathrm{~min}$ at $72^{\circ} \mathrm{C}$, followed by final extension at $72^{\circ} \mathrm{C}$ for 10 min. PCR products were visualized on $1.5 \%$ agarose gel, purified using an $\mathrm{ENZA}^{\mathrm{TM}}$ gel extraction kit (Omega, Norcross, GA, USA), and then cloned into the pMD19-T vector (TaKaRa, Shiga, Japan) according to manufacturer instructions. All samples were sequenced by the Beijing Genomics Institute (Beijing, China).

\section{Bioinformatics analysis of $\mathrm{HbSINA4}$}

Databases such as NCBI (http://ncbi.nlm.nih.gov/), ExPAsy (http://www.expasy.org/ tools/pitool.html), TMHMM (http://www.cbs.dtu.dk/services/TMHMM/), TargeP (http:// www.cbs.dtu.dk/services/SignalP), and PSORT (http://psort.hgc.jp/form.html), were used to analyze the sequences of HbSINA4. Based on the predicted HbSINA4 amino acid sequence, 
the package MEGA5.1 was used for amino acid sequence homology comparison analysis and to construct the evolutionary tree.

\section{RT-PCR analysis of HbSINA4}

RT-PCR was conducted using the SYBR Premix Ex TaqTM Kit, and the quantitative primers [A2-FP (TGGGTGACATCAGGTGGTGTTTGG) and A2-RP (GAGCATTCAGATC CCGCATAA)] were also designed according to the HbSINA4 sequence. $\beta$-Tublin was used as a reference gene [A3-FP (CCAAGTTCTGGGAGGTGATCTG) and A3-RP (TTGTAGTAGA CGTTGATGCGCTC)]. RT-PCR data was analyzed using the double standard curve method as described by Zhang et al. (2005).

\section{RESULTS}

\section{HbSINA4 cloning and bioinformatics analysis}

A cDNA of 1052 bp was amplified successfully (Figure 1). Using the ORF finder procedure in NCBI, the 5'- and 3'-untranslated regions were 82 and $1082 \mathrm{bp}$ in length, respectively. The open reading frame of this gene was $771 \mathrm{bp}$, coding for 256 amino acids (Figure 2). The predicted molecular weight of this polypeptide was $29.53 \mathrm{kDa}$ and the isoelectric point was 8.32. Prosite Scan analysis indicated that this polypeptide included the family signature motifs of zinc finger SIAH-type profile (single underline in Figure 2), 6 protein kinase $\mathrm{C}$ phosphorylation sites (bold in Figure 2), $4 \mathrm{~N}$-nutmeg acylation sites (wave underline in Figure 2), 2 N-glycosylation sites (italics in Figure 2), amidation site (double underline in Figure 2), and 2 casein kinase II phosphorylation sites (dotted underline in Figure 2). The TMHMM database revealed that this protein did have the membrane transfer function. Signal IP3.0 analysis revealed that this protein did not have a signal peptide, indicating that it is a non-secretory protein. PSORT subcellular localization analysis showed that this protein was located in the cytoplasm.

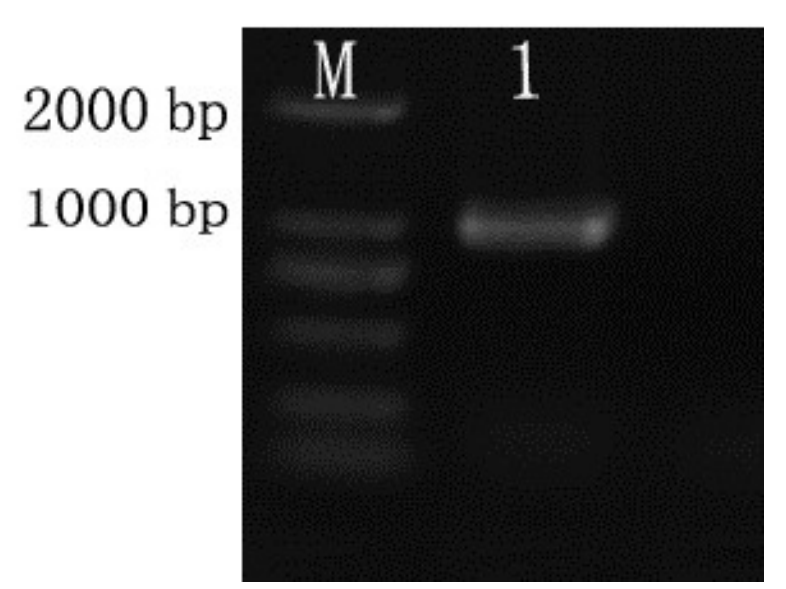

Figure 1. Amplification of the HbSINA4 gene from Tibetan hulless barley. Lane M=DL2000; lane 1=HbSINA4. 


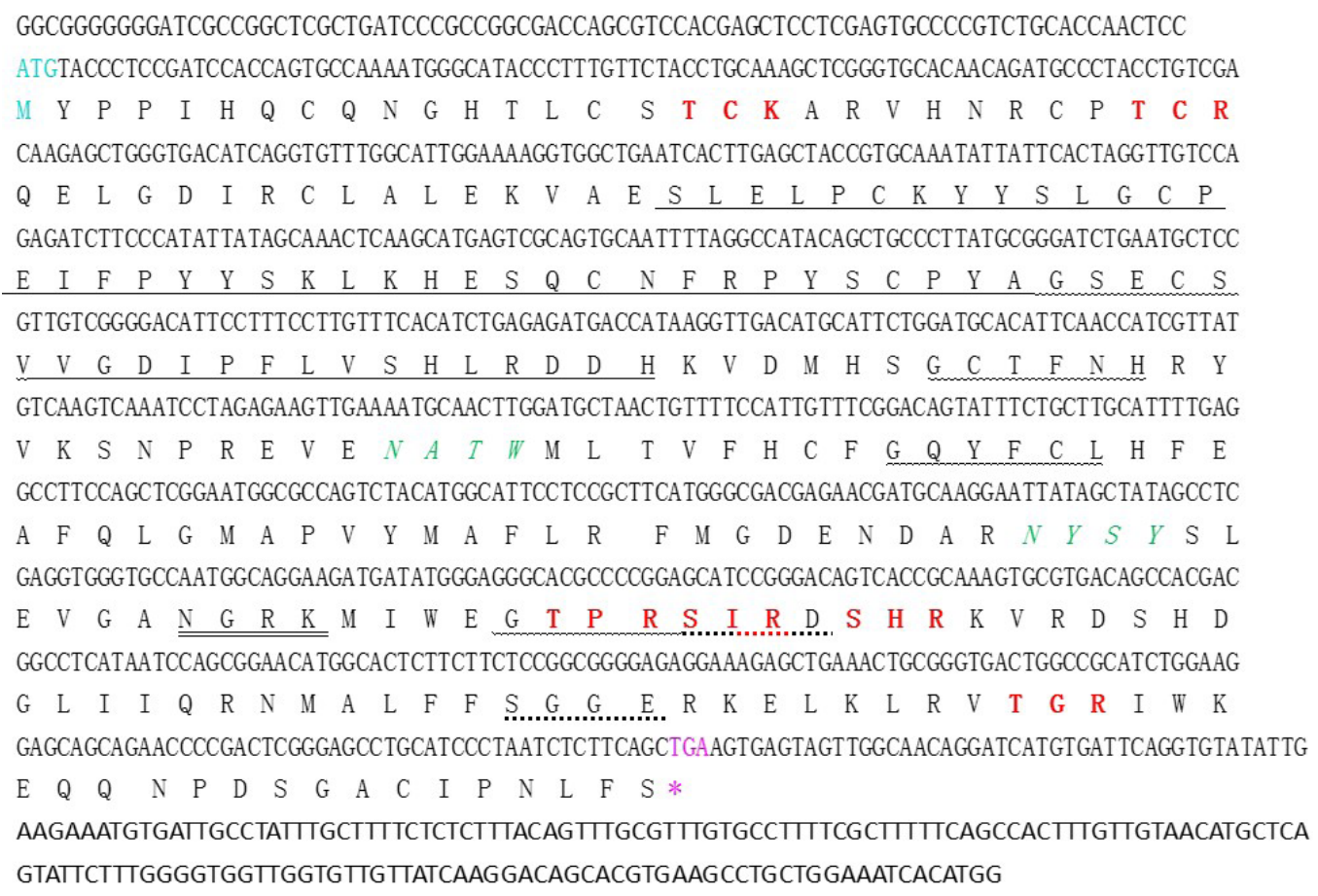

Figure 2. Nucleotide and predicted amino acid sequences of the HbSINA4 gene (single underline: zinc finger SIAH-type profile family signature residuals; bold: proteinase $\mathrm{C}$ phosphorate site; wavy line: N-myristoylation site; italic: N-glycosylation site; double line: amidation site; dashed line: casein kinase II phosphorylation sites).

BLASTn analysis indicated that this sequence of SINA had high similarity (90-96\%) to SINA found in O. sativa, millet, maize, Brachypodium distachyon, Solamum lycopersicum, beans, Solanum tuberosum, and string beans. Interfering amino acid sequence homology, analysis (Figure 3) suggested a similarity of $92-98 \%$ between HbSINA4 and the orthologs in these species. Based on the amino acid sequence, we built a phylogenetic tree, which revealed consistent evolution between HbSINA4 and its orthologs in other plants. HbSINA4 showed a close relationship with the SINA family in millet, $B$. distachyon, and $O$. sativa, whereas the relationship was less close to the SINA family in S. tuberosum and S. lycopersicum. The complete SINA gene was cloned successfully in highland barley, named HbSINA4, and recorded given the accession No. KJ699391 in GenBank.

\section{Expression patterns of the $\mathrm{HbSINA4}$ gene}

The expression patterns of HbSINA4 are presented in Figure 4. When the RSMC reached $33.4 \%$, the expression of HbSINA4 was up-regulated. With decreasing RSMC, gene expression also decreased. When the soil moisture content $(\mathrm{C} 4)$ reached a normal level, expression was up-regulated. During drought stress, expression decreased when RSMC was drastically decreased. After $2 \mathrm{~h}$ water recovery, expression was again up-regulated over time. After $8 \mathrm{~h}$, the expression got the pink when RSMC reached $33.4 \%$. These results indicate that 
the expression of HbSINA4 was induced by drought stress and may be related to the drought resistance response in barley.

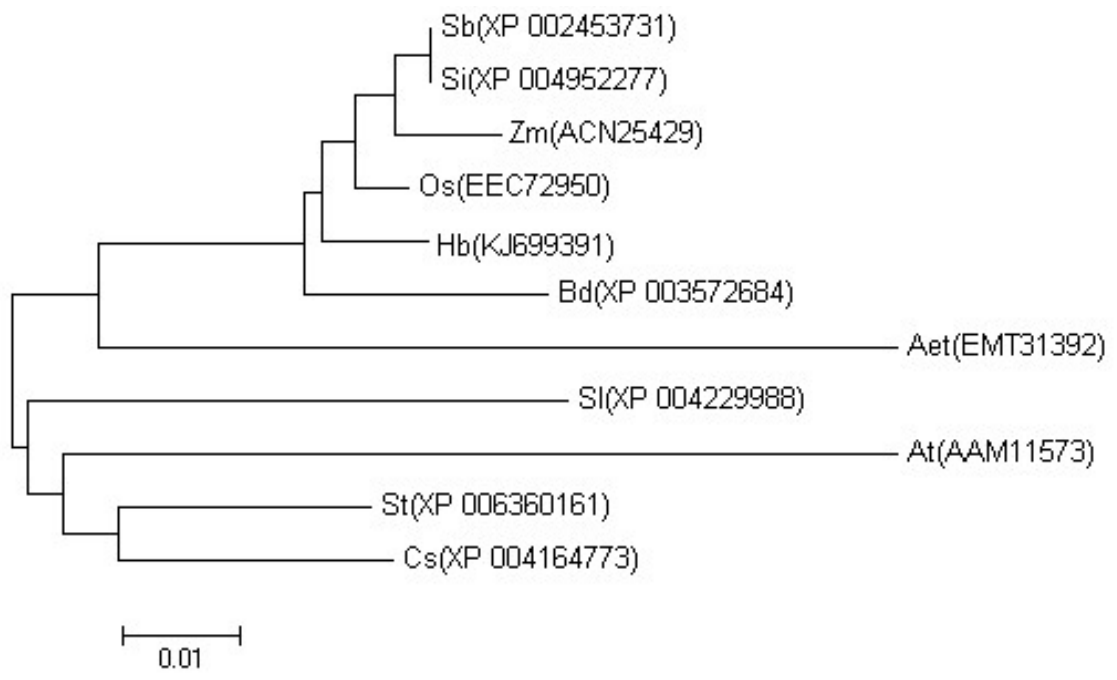

Figure 3. Phylogenetic tree of HbSINA4 and other SINA from different genera. $\mathrm{Sb}=$ Sorghum bicolor; $\mathrm{Si}=$ Setaria italica; $\mathrm{Os}=$ Oryza sativa $; \mathrm{Zm}=$ Zea mays $; \mathrm{Cs}=$ Cucumis sativus; $\mathrm{Bd}=$ Brachypodium distachyon $; \mathrm{S} 1=$ Solamum lycopersicum; $\mathrm{At}=$ Arabidopsis thaliana $; \mathrm{St}=$ Solanum tuberosum; $\mathrm{Hb}=$ Tibetan hulless barley; $\mathrm{Aet}=$ Aegilops tauschii .

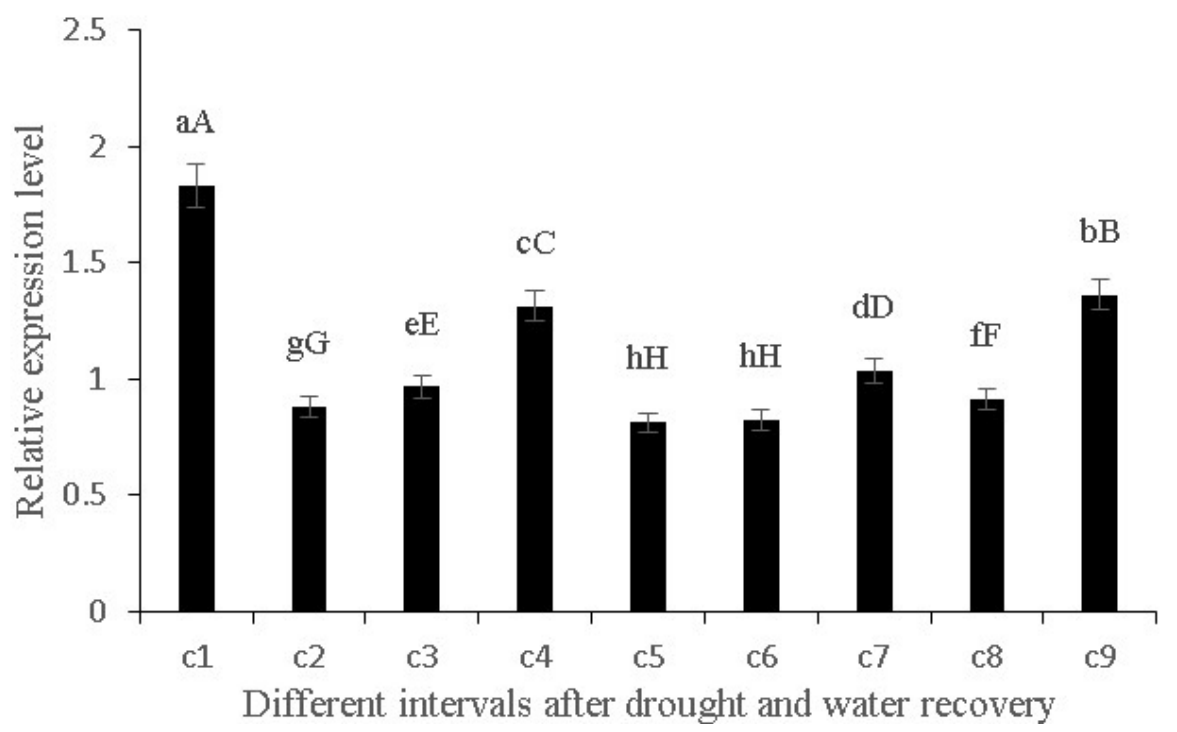

Figure 4. Real-time PCR analysis of the HbSINA4 gene expression at different intervals during drought treatments. Drought stress at 6 levels, c1, 33.4\%; c2, 27.5\%; c3, 21.1; c4, 15.5\%; c5, 9.8\%; and c6, 4.8\%, was examined. Watering recovery was tested at c7, $2 \mathrm{~h} ; \mathrm{c} 8,4 \mathrm{~h}$; and c9, $8 \mathrm{~h}$. One-way analysis of variance and the Duncan multiple range test were carried out, and the different lower case letter indicates significance at $\mathrm{P}=0.05$, while the different upper case letters indicate significance at $\mathrm{P}=0.01$. 


\section{DISCUSSION}

SINA was first identified in Drosophila melanogaster (Carthew and Gubin, 1999) and represents an important gene family in plants. There are 5 members in Populus alba, 6 members in O. sativa, more than 6 members in Zea mays, and 2 members in Bryophytes (Wang, 2007). However, its function and biological process remain unclear. SINA has been shown to function as an E3 (Xie et al., 2002). All SINA in plant contain a SINA domain and a RING domain, and the 2 domains are very conserved. Based on evolution analysis, plant SINA proteins are placed into 2 groups. Most group I proteins contain a motif 3 conserved domain at the C-terminus, while no group II proteins have this motif (Wang, 2007). Based on Arabidopsis transgenic plants, 2 members in group I were found to increase the length of the taproot, but reduce the distribution of the lateral root (Wang, 2007). In contrast, group II plants can reduce the length of the taproot. SINAT5 in Arabidopsis has been thoroughly examined. As a single subunit, SINAT5 can degrade NAC1, which is an important factor in lateral root formation. Over-expressed SINAT5 reduces lateral root growth of Arabidopsis, whereas a mutant from conserve Cys in over-expressed $R I N G$ domain will increase lateral root growth (Xie et al., 2002). Down-regulation of SINAT5 allows the formation of lateral roots in Arabidopsis. Additionally, Fraire-Velázquez and Lozoya-Gloria (2003) obtained a cDNA that was very similar to SINAT5 in bean. This gene may be induced by pathogenic bacteria and may play roles in the disease resistance response. Jaradat (1999) identified the GhSINAH-1 gene, which is related to cotton fiber cell elongation. Colligating with the achievements of this gene, SINA in plants differ in functions, and may be involved in different physiological processes. However, these functions are not well-understood, with exception of SINAT5 in Arabidopsis.

This study was based on previously unpublished results from transcriptome sequencing of barley under different drought stress conditions. In this study, we cloned HbSINA4, which was found to belong to group I because its motif 3 is conserved at the C-terminus. RTPCR analysis indicated that HbSINA4 expression changed in response to drought stress, and thus may play a very important role in drought resistance. Furthermore, this result suggested that SINA has diverse functions in plant. This is the first report to demonstrate that SINA is involved in drought stress. It remains unclear whether the response to drought is related to the regulation of the taproot and lateral root in Arabidopsis. In addition, it is unknown whether HbSINA4 is involved in other physical processes. Therefore, further studies are needed to clarify the role of HbSINA4 in plants.

\section{ACKNOWLEDGMENTS}

Research supported by the Special Funds for Preliminary Research of "973" Plan (\#2012CB723006), the National Scientific and technological Support Plan (\#2012BAD03B01), and the Specific Financial Funds in Tibet Autonomous Region (\#2011XZCZZX001).

\section{REFERENCES}

Acar O, Tiirkan I and Özdemir F (2001). Superoxide dismutase and peroxidase activities in drought sensitive and resistant barley (Hordeum vulgate L.) varieties. Acta Physiol. Plant 23: 351-356.

Boyer JS (1982). Plant productivity and environment. Science 218: 443-448.

Carthew R and Gubin GM (1999). Seven in absentia, a gene required for specification of R7 cell fate in the Drosophila 
eye. Cell 63: 561-577.

Den Herder G, De Keyser A, De Rycke R, Rombauts S, et al. (2008). Seven in absentia proteins affect plant growth and nodulation in Medicago truncatula. Plant Physiol. 148: 369-382.

Diab AA, Teulat-Merah B, This D, Ozturk NZ, et al. (2004). Identification of drought-inducible genes and differentially expressed sequence tags in barley. Theor. Appl. Genet. 109: 1417-1425.

Fraire-Velázquez S and Lozoya-Gloria E (2003). Different early gene expression in Phaseolus vulgaris to Mexican isolates of Colletotrichum lindemuthianum in incompatible interactions. Physiol. Mol. Plant P. 63: 79.

Guo PG, Baum M, Grando S, Ceccarelli S, et al. (2009). Differentially expressed genes between drought-tolerant and drought-sensitive barley genotypes in response to drought stress during the reproductive stage. J. Exp. Bot. 60: 3531-3544.

Jaradat TT (1999). Studies of cotton cDNAs encoding a seven in absentia homolog, a potential DNA-binding, and a cell wall protein. Doctoral thesis. Department of Biological Science of Texas Tech University, Lubbock.

Nie YY (2009). Drought tolerance QTL fine mapping in rice. Wuhan: Huazhong Agricultural University, Plant Science and Technology. Available at [http://www.cnki.net/KCMS/detail/detail.aspx].

Oukarroum A, Madidi SE, Schansker G and Strasser RJ (2007). Probing the responses of barley cultivars (Hordeum vulgare L.) by chlorophyll a fluorescence OLKJIP under drought stress and re-watering. Environ. Exp. Bot. 60: 438-446.

Singh TN, Aspinall D and Paleg LG (1972). Proline accumulation and varietal adaptability to drought in barley: a potential metabolic measure of drought resistance. Nat. New Biol. 236: 188-190.

Wang M (2007). Plant SINA gene families evolution and expression analysis and SINA gene function in Arabidopsis thaliana. Beijing: China Agricultural University, Institute of Agriculture and Biotechnology. Doi: 10.7666/d. y1108525.

Wang W, Vinocur B and Altman A (2003). Plant responses to drought, Salinity and extreme temperatures: towards genetic engineering for stress tolerance. Planta 218: 1-14.

Wang YT (2013). Sorghum drought resistance mechanism and their evaluation index. Shenyang: Shenyang Agricultural University, Agricultural College. Available at [http://www.cnki.net/KCMS/detail/ detail.aspx].

Welsch R, Maass D, Voegel T, Dellapenna D, et al. (2007). Transcription factor RAP2.2 and its interacting partner SINAT2: stable elements in the carotenogenesis of Arabidopsis leaves. Plant Physiol. 145: 1073-1085.

Xie Q, Guo HS, Dallman G, Fang S, et al. (2002). SINAT5 promotes ubiquitin-related degradation of NACL to attenuate auxin signals. Nature 419: 167-170.

Zhang CY, Xu SG and Huang XX (2005). A novel and convenient relative quantitative method of fluorescence real time RT-PCR assay based on slope of standard curve. Prog. Biophys. Mol. Biol. 32: 883-888.

Zhang H, Song GQ, Ji WQ and Hu YG (2009). Gene induction by drought stress in wheat variety xiaoyan 22 and their expression analysis. J. Agric. Biotechnol. 17: 670-676.

Zhao KF and Li FZ (1999). Chinese halophytes. Science Press, Beijing, 28-29.

Zhao XH, Zhang XC, Li SS, Li LH, et al. (2005). QTL mapping of physiological traits of wheat relating to drought resistance. Acta Bot. Boreali Occid. Sin. 4: 696-699. 